\title{
CODAZZI-TENSOREN UND KENNZEICHNUNGEN SPHÄRISCHER IMMERSIONEN
}

\author{
BERND WEGNER
}

Durch Berechnung der Laplaceschen des Quadrates der Länge des zweiten Fundamental tensors einer minimalen Immersion in einen Raum konstanter Krümmung haben J. Simons [9], S. S. Chern, M. do Carmo und S. Kobayashi [4] sowie andere Autoren Integralformeln erhalten, mit denen sich gewisse minimale Immersionen in Sphären charakterisieren lassen. K. Yano und S. Ishihara [12] haben die Berechnung dieser Laplaceschen für isometrische Immersionen mit lokal parallelisierbarem Normalenbündel durchgeführt und ebenfalls starke Charakterisierungssätze erhalten. Bei S. Braidi und C. C. Hsiung [1] wird der Fall beliebiger isometrischer Immersionen in Räume konstanter Krümmung diskutiert.

Die Formel von K. Yano und S. Ishihara weist Analogien zu einer Formel von K. Nomizu und B. Smyth [8] für die Kodimension 1 auf, die inzwischen von B. Smyth [10] für höhere Kodimensionen unter der Voraussetzung verallgemeinert wurde, daß die Immersion einen parallelen Schnitt $\xi$ im Normalenbündel zuläßt, in dessen Richtung die Spur des zweiten Fundamentaltensors konstant ist. Die Formel von B. Smyth hat eine recht einfache Gestalt und besitzt eine Reihe interessanter Anwendungen, denen in dieser Arbeit einige neue hinzugefügt werden sollen. Ferner soll unter Abänderung der Beweisführung von Smyth gezeigt werden, daß man eine analoge Formel für die Laplacesche des Quadrates der Länge eines $(1,1)$-Tensorfeldes beweisen kann, wenn das Tensorfeld Codazzi-Gleichungen erfüllt und seine Spur konstant ist. Ist dieses Feld invertierbar, so erhält man aus der allgemeinen Formel durch Übertragung einer aus der Hyperflächentheorie bekannten Ummetrisierung eine Formel für die Laplacesche des Quadrates der Länge des inversen Tensorfeldes. Während die Formel von Smyth Anwendungen auf Immersionen und Normalvektorfelder $\xi$ mit konstanter mittlerer Krümmung in Richtung $\xi$ erlaubt, liefert diese zweite Formel analoge Ergebnisse unter der Voraussetzung, daß die Summe der Hauptkrümmungsradien in Richtung $\xi$ konstant ist.

In dieser Arbeit werden unter den Anwendungen der genannten Formeln Kennzeichnungen von sphärischen Immersionen bzw. Produkten sphärischer Immersionen diskutiert, die Ergebnisse von B. Smyth [10], K. Yano und S. Ishihara [12], B. Y. Chen [2] und vom Verf. selbst [11] ergänzen oder verallge-

Communicated by W. P. A. Klingenberg, September 5, 1972. 
meinern. Es sei an dieser Stelle vermerkt, daß der Anstoß zu der vorliegenden Arbeit aus der Arbeitsgemeinschaft Differentialgeometrie an der TU Berlin kam, deren Mitgliedern ich hiermit für ihre wertvollen Diskussionsbeiträge danke.

\section{Verallgemeinerung der Formel von Smyth}

Inhalt der folgenden Ausführungen werden Formulierung und Beweis der verallgemeinerten Formel (1.10) von Smyth [10] sein. Der Beweis wird sich darauf beschränken, den Beweisteil anzugeben, der nicht zu den Ausführungen in [8] und [10] analog verläuft, und die Stellen zu markieren, an denen die angegebenen Voraussetzungen benötigt werden. Zuvor sollen jedoch einige Bezeichnungen vereinbart werden: $M$ sei eine hinreichend oft differenzierbare Riemannsche Mannigfaltigkeit der Dimension $n$ mit Metrik $\langle.,$.$\rangle und Norm$ $\|\|,$.$D der dadurch bestimmte Riemannsche Zusammenhang (kovariante$ Ableitungsoperator), $R$ der Krümmungstensor. Mit grad werde der Gradientenoperator bezeichnet, mit div der Divergenzoperator, mit $\Delta$ der LaplaceOperator und mit Sp die Spurbildung. Für Grundlagen wird auf das Buch von S. Kobayashi und K. Nomizu [6] verwiesen.

Definition. Sei $\boldsymbol{A}$ ein differenzierbares $(1,1)$-Tensorfeld über $M$; als Feld von linearen Transformationen der Tangentialfasern von $M$ sei $A$ selbstadjungiert; ferner erfülle $A$ für jeden Punkt $p$ von $M$ und für jedes Paar $X, Y$ von Tangentialvektoren an $M$ in $p$ Codazzigleichungen

$$
\left(D_{X} A\right)(Y)=\left(D_{Y} A\right)(X)
$$

Dann heißt $A$ Codazzi-Tensor (feld) über $M$.

Bemerkung 1. Beispiele für Codazzi-Tensoren gibt es in der Theorie der isometrischen Immersionen in Räume konstanter Krümmung. Ist nämlich $\xi$ ein paralleler Schnitt im Normalenbündel der Immersion, so definiert die Tangentialprojektion $-A_{\xi}$ des kovarianten Differentials von $\xi$ (im Oberraum) einen Codazzi-Tensor über dem Definitionsbereich des Schnittes. Ferner ist jeder lokal konform flachen Riemannschen Mannigfaltigkeit in natürlicher Weise ein Codazzi-Tensor zugeordnet.

Satz 1. Für einen Codazzi-Tensor $A$ über $M$ gilt die folgende Formel:

$$
\begin{aligned}
\frac{1}{2} \Delta \operatorname{Sp}\left(A^{2}\right)= & \sum_{i<j}\left(\lambda_{i}-\lambda_{j}\right)^{2} K_{i j}+\|D A\|^{2} \\
& -\|\operatorname{grad} \operatorname{Sp}(A)\|^{2}+\operatorname{div}(A(\operatorname{grad} \operatorname{Sp}(A))),
\end{aligned}
$$

dabei sind die $\lambda_{i}$ die Eigenwerte von $A$ für eine Orthonormalbasis von Eigenrichtungen $E_{i}$ von $A$, und mit $K_{i j}$ wird die Schnittkrümmung von $M$ in Richtung $E_{i}$ und $E_{j}$ bezeichnet.

Beweis. Für einen Tangentialvektor $X$ an $M$ gilt 


$$
X\left(\operatorname{Sp}\left(A^{2}\right)\right)=2 \operatorname{Sp}\left(D_{X} A \circ A\right) .
$$

Sei $p \in M$ und $E_{i}(i=1, \cdots, n)$ ein differenzierbares System von orthonormierten Vektorfeldern über einer Umgebung von $p$, so daß die $E_{i}$ in $p$ Eigenrichtungen der selbstadjungierten Transformation $A$ sind und ferner an der Stelle $p D E_{i}=0$ gilt. Mit (1.3) erhält man in $p$

$$
\frac{1}{2} \Delta \operatorname{Sp}\left(A^{2}\right)=\sum_{j=1}^{n} \operatorname{Sp}\left(\left(D_{E_{j}}\left(D_{E_{j}} A\right)\right) \circ A\right)+\|D A\|^{2} .
$$

Wird der Tangentialvektor $X$ an $M$ in $p$ zu einem ebenfalls mit $X$ bezeichneten differenzierbaren Vektorfeld fortgesetzt, so daß in $p D X=0$ gilt, so folgt mit den Codazzigleichungen (1.1) in $p$

$$
\left(D_{E_{j}}\left(D_{E_{j}} A\right)\right)(X)=D_{X}\left(\left(D_{E_{j}} A\right)\left(E_{j}\right)\right)+\left[R\left(E_{j}, X\right), A\right]\left(E_{j}\right),
$$

wobei mit [., . .] die Komutator-Klammer linearer Abbildungen bezeichnet wird. Damit erhält (1.4) die Gestalt

$$
\begin{aligned}
\frac{1}{2} \Delta \operatorname{Sp}\left(A^{2}\right)= & \sum_{i, j=1}^{n}\left\langle\left[R\left(E_{j}, A\left(E_{i}\right)\right), A\right]\left(E_{j}\right), E_{i}\right\rangle \\
& +\sum_{i, j=1}^{n}\left\langle D_{A\left(E_{i}\right)}\left(\left(D_{E_{j}} A\right)\left(E_{j}\right)\right), E_{i}\right\rangle+\|D A\|^{2} .
\end{aligned}
$$

Ebenfalls durch Anwendung von (1.1) in $p$ bekommt man

$$
\sum_{j=1}^{n}\left(D_{E_{j}} A\right)\left(E_{j}\right)=\operatorname{grad} \operatorname{Sp}(A)
$$

und deshalb

$$
\begin{aligned}
& \sum_{i, j=1}^{n}\left\langle D_{A\left(E_{i}\right)}\left(\left(D_{E_{j}} A\right)\left(E_{j}\right)\right), E_{i}\right\rangle \\
& \quad=-\|\operatorname{grad} \operatorname{Sp}(A)\|^{2}+\operatorname{div}(A(\operatorname{grad} \operatorname{Sp}(A)))
\end{aligned}
$$

Wird mit $\lambda_{i}$ der Eigenwert von $A$ in $p$ in Richtung $E_{i}$ bezeichnet, so liefert die Definition der Schnittkrümmung sofort

$$
\begin{aligned}
& \sum_{i, j=1}^{n}\left\langle\left[R\left(E_{j}, A\left(E_{i}\right)\right), A\right]\left(E_{j}\right), E_{i}\right\rangle \\
& \quad=\sum_{i, j=1}^{n}\left(\lambda_{i} \lambda_{j}-\lambda_{i}^{2}\right)\left\langle R\left(E_{j}, E_{i}\right) E_{j}, E_{i}\right\rangle=\sum_{i<j}\left(\lambda_{i}-\lambda_{j}\right)^{2} K_{i j} .
\end{aligned}
$$

Aus (1.6), (1.8) und (1.9) folgt dann (1.2).

Folgerung 1. Für einen Codazzi-Tensor $A$ über $M$, dessen Spur konstant ist, gilt die folgende Formel: 


$$
\frac{1}{2} \Delta \operatorname{Sp}\left(A^{2}\right)=\sum_{i<j}\left(\lambda_{i}-\lambda_{j}\right)^{2} K_{i j}+\|D A\|^{2} .
$$

Bemerkung 2. Eine Berechnung von $\Delta \mathrm{Sp}\left(A^{2}\right)$ wurde unabhängig (etwa zu gleicher Zeit) von U. Simon (unpubliziert) in lokalen Koordinaten durchgeführt. Auf Konsequenzen der Formel von Simon wird hier nicht eingegangen. Ferner hat H. F. Münzner Formeln für die Laplacesche der elementarsymmetrischen Funktionen der Eigenwerte von $A$ aufgestellt (mündliche Mitteilung in Oberwolfach, bisher unpubliziert). Die Formel (1.10) wurde von B. Smyth in [10] für die unter Bemerkung 1 erwähnten Codazzi-Tensoren $A_{\xi}$ bei Voraussetzung einer konstanten Spur abgeleitet. Dabei spielt dort der Zielraum der Immersion für den Nachweis der Gleichung (1.9) eine gewisse Rolle, die jedoch leicht umgangen werden kann, wie man hier sieht.

\section{Invertierbare Codazzi-Tensoren}

Die folgenden Betrachtungen werden zeigen, daß es neben der Formel von Smyth weitere sinnvolle Spezialisierungen von (1.2) bzw. (1.10) gibt. Das Verfahren dafür ist eine Übertragung der aus der Theorie der Eiflächen bekannten Ummetrisierung durch die dritte Fundamentalform.

Es sei $A$ ein Codazzi-Tensor über der Riemannschen Mannigfaltigkeit $M$ mit der Metrik $\langle.,$.$\rangle . Es werde zusätzlich vorausgesetzt, daß A$ invertierbar ist, d. $h$. , Det $(A) \neq 0$ gilt. Dann wird durch

$$
\tilde{\langle X}, Y \overline{\rangle}=\langle A(X), A(Y)\rangle
$$

eine weitere Riemannsche Metrik $\widetilde{<} ., . \tilde{>}$ auf $M$ definiert. Alle Größen, die zu dieser neuen Metrik gehören, werden mit einer Schlange versehen.

Lemma 1. Zwischen den Riemannschen Zusammenhängen $D$ und $\tilde{D}$ von $\langle.,$.$\rangle und \widetilde{\zeta}, .,$.$\rangle besteht die folgende Relation:$

$$
\tilde{D}_{X} Y=\left(A^{-1} \circ D_{X} \circ A\right)(Y) \text {. }
$$

Der Beweis von Lemma 1 erfolgt durch einfaches Nachprüfen der Axiome für den Riemannschen Zusammenhang von $\tilde{\langle} ., . \tilde{\rangle}$ für die rechte Seite von (2.2). Die Codazzi-Eigenschaft von $A$ wird benötigt, um die Torsionsfreiheit von $A^{-1} \circ D \circ A$ nachzuweisen (vgl. N. Hicks, Linear perturbations of connexions, Mich. Math. J. 12 (1965) 389-397). Ebenso leicht erhält man

Lemma 2. $A^{-1}$ ist ein Codazzi-Tensor über der Riemannschen Mannigfaltigkeit $(M, \widetilde{\zeta} ., . \tilde{\rangle})$, d. h., es gilt

$$
\left(\tilde{D}_{X} A^{-1}\right)(Y)=\left(\tilde{D}_{Y} A^{-1}\right)(X)
$$

für beliebige Tangentialvektoren $X, Y$ an einen Punkt von $M$.

Wegen (2.3) kann man nun die Formel (1.2) für $A^{-1}$ formulieren. Für geometrische Anwendungen ist es jedoch interessant, einige dabei auftretende 
Größen in der Ausgangs-Metrik zu interpretieren. Dazu leitet man aus (2.1) und (2.2) die folgenden Relationen ab:

$$
\begin{gathered}
\widetilde{\mathrm{Sp}}\left(A^{-1}\right)=\mathrm{Sp}\left(A^{-1}\right), \quad \widetilde{\mathrm{Sp}}\left(A^{-2}\right)=\operatorname{Sp}\left(A^{-2}\right), \\
\tilde{R}(X, Y) Z=A^{-1}(R(X, Y) A(Z)), \\
\tilde{K}_{i j}=\left(\lambda_{i} \lambda_{j}\right)^{-1} K_{i j} .
\end{gathered}
$$

Mit (2.4) und (2.6) erhält man aus (1.2) bzw. (1.10) die

Folgerung 2. Ist $A$ ein invertierbarer Codazzi-Tensor über $M$, so gilt mit den vorangegangenen Bezeichnungen

$$
\begin{aligned}
\frac{1}{2} \tilde{\Delta} \operatorname{Sp}\left(A^{-2}\right)= & \sum_{i<j} \frac{K_{i j}}{\lambda_{i} \lambda_{j}}\left(\frac{1}{\lambda_{i}}-\frac{1}{\lambda_{j}}\right)^{2}+\tilde{\|} \tilde{D} A^{-1} \tilde{\|}^{2} \\
& -\| \widetilde{\operatorname{grad}} \operatorname{Sp}\left(A^{-1}\right) \tilde{\|}^{2}+\widetilde{\operatorname{div}}\left(A^{-1}\left(\widetilde{\operatorname{grad}} \operatorname{Sp}\left(A^{-1}\right)\right)\right),
\end{aligned}
$$

und für den Fall, daß die Spur von $A^{-1}$ konstant ist,

$$
\frac{1}{2} \tilde{\Delta} \mathrm{Sp}\left(A^{-2}\right)=\sum_{i<j} \frac{K_{i j}}{\lambda_{i} \lambda_{j}}\left(\frac{1}{\lambda_{i}}-\frac{1}{\lambda_{j}}\right)^{2}+\tilde{\|} \tilde{D} A^{-1} \tilde{\|}^{2} .
$$

Bevor Konsequenzen aus (1.10) und (2.8) diskutiert werden, sollen noch einige Betrachtungen zur obigen Ummetrisierung im Fall isometrischer Immersionen in euklidische Räume angestellt werden. Sei $x: M \rightarrow R^{N}$ eine hinreichend oft differenzierbare isometrische Immersion der $n$-dimensionalen geschlossenen Riemannschen Mannigfaltigkeit $M$. Das Normalenbündel von $x$ lasse einen globalen parallelen Schnitt $\xi \mathrm{zu}$, so daß der entsprechende Codazzi-Tensor $A_{\xi}$ (vgl. Bemerkung 1) invertierbar ist. Wird mit $\xi_{r}(r=1, \cdots, N-n)$ ein lokales Orthonormalsystem im Normalenbündel von $x$ bezeichnet, so gibt $\xi$ analog zur Konstruktion der mittleren Krümmungsnormale Anlaß zu einer Reihe von globalen Normalvektorfeldern

$$
\eta(k, \xi)=\frac{1}{n} \sum_{r=1}^{N-n} \operatorname{Sp}\left(A_{\xi r} \circ A_{\xi}^{k}\right) \xi_{r} \quad(k \in Z) .
$$

Man macht sich leicht klar, daß diese Definition von der Wahl der $\xi_{r}$ unabhängig ist.

Für $k=0$ erhält man in (2.9) die mittlere Krümmungsnormale von $x$. Für $k=1$ liefert (2.9) die mittlere Krümmungsnormale von $\xi$, wenn $\xi$ als isometrische Immersion der nach (2.1) ummetrisierten Mannigfaltigkeit $M$ in den $R^{N}$ betrachtet wird. Insbesondere folgt, daß bei kompaktem $M \eta(-1, \xi)$ nicht identisch verschwinden kann, da es keine minimalen Immersionen kompakter Mannigfaltigkeiten in euklidische Räume gibt.

Die vorangegangenen Betrachtungen führen nun auf die folgende Charakterisierung sphärischer Immersionen durch eine Relation zwischen der Summe 
der Hauptkrümmungsradien in Richtung $\xi$ und dem Stützabstand in Richtung $\eta(-1, \xi)$, deren Analogon für den Stützabstand in Richtung $\eta(0, \xi)$ schon lange bekannt ist (vgl. u.a. [3]):

Satz 2. Für eine isometrische Immersion $x: M \rightarrow R^{N}$ der geschlossenen Mannigfaltigkeit $M$ mit parallelem Schnitt $\xi$ im Normalenbündel gelte $\operatorname{Det}\left(A_{\xi}\right)$ $\neq 0$ und

$$
\frac{1}{n} \operatorname{Sp}\left(A_{\xi}^{-1}\right)+\langle x, \eta(-1, \xi)\rangle \leq 0(\geq 0),
$$

$\left(\langle.,\right.$.$\left.\rangle Standard-Metrik für den R^{N}\right)$. Dann liegt $x(M)$ auf einer Sphäre.

Beweis. $\quad M$ werde durch $A_{\xi}$ gemäß (2.1) ummetrisiert. Für die differenzierbare Funktion $f=\langle x, \xi\rangle$ auf $M$ gilt dann mit einem geeignet fortgesetzten $\tilde{\langle}$. ,.$\overline{\rangle}$-Orthonormalsystem $\tilde{E}_{i}$ von Eigenvektoren von $A_{\xi}$ mit Eigenwerten $\lambda_{i}$ in $p \in M$ wegen der Parallelität von $\xi$ :

$$
\begin{aligned}
\tilde{\Delta} f & \left.=-\sum_{i=1}^{n} \tilde{\langle} \tilde{D}_{\tilde{E}_{i}}\left(A_{\xi}^{-1}\left(x^{T}\right)\right), \tilde{E}_{i}\right\rangle=-\sum_{i=1}^{n}\left\langle D_{\tilde{E}_{i}} x^{T}, A_{\xi}\left(\tilde{E}_{i}\right)\right\rangle \\
& =-\sum_{i=1}^{n} \lambda_{i}^{-1}-\sum_{i=1}^{n} \lambda_{i}^{-1}\left\langle x^{\perp}, \bar{D}_{A_{\xi}\left(\tilde{E}_{i}\right)} A_{\xi}\left(\tilde{E}_{i}\right)\right\rangle \\
& =-\operatorname{Sp}\left(A_{\xi}^{-1}\right)-\langle x, n \eta(-1, \xi)\rangle,
\end{aligned}
$$

wenn mit $x^{T}$ bzw. $x^{\perp}$ Tangential-bzw. Normalanteil von $x$ und mit $\bar{D}$ der Riemannsche Zusammenhang des $R^{N}$ bezeichnet wird. Man beachte, da $\beta$ $A_{\xi}\left(\tilde{E}_{i}\right)(i=1, \cdots, n)$ ein $\langle.,$.$\rangle -Orthonormalsystem ist. Aus der Kompaktheit$ von $M$ und aus (2.10) folgt mit (2.11) nach dem Lemma von Hopf, daß $f$ konstant ist. Da $\xi$ parallel ist, gilt dann für eine Eigenrichtung $E_{i}$ von $A_{\xi}$

$$
0=E_{i}(f)=\left\langle x, \bar{D}_{E_{i}} \xi\right\rangle=-\lambda_{i}\left\langle x, E_{i}\right\rangle .
$$

Wegen $\lambda_{i} \neq 0$ ist damit $\left\langle x, E_{i}\right\rangle=0$, also $E_{i}\left(\|x\|^{2}\right)=0$ für alle Eigenrichtungen $E_{i}$. Damit ist $\|x\|$ konstant.

Bemerkung 3. Für die Kodimension 1 liefert (2.11) gerade eine der klassischen Minkowski-Formeln.

Analog zum Beweis von Lemma 1 und 2 bei D. Ferus [5] (vgl. auch [10]) verläuft über den Satz von Riemann-Roch nach der Ummetrisierung (2.1) der Beweis von

Satz 3. Sei $x: S^{2} \rightarrow R^{N}$ eine isometrische Immersion einer geschlossenen 2-Mannigfaltigkeit vom Geschlecht 0 , deren Normalenbündel einen parallelen Schnitt $\xi$ mit $\operatorname{Det}\left(A_{\xi}\right) \neq 0$ und $\operatorname{Sp}\left(A_{\xi}^{-1}\right)=$ const. zuläßt. Dann liegt $x\left(S^{2}\right)$ in

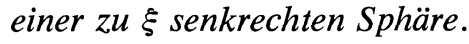




\section{Kennzeichnungen sphärischer Immersionen}

Ein wichtiges Hilfsmittel bei der Vereinfachung der Beweise der nun folgenden Kennzeichnungen sphärischer Immersionen durch parallele Schnitte im Normalenbündel ist eine Variante des de Rhamschen Zerlegungssatzes, die von R. Walden bewiesen wurde (bisher unpubliziert):

Lemma 3. Sei $A$ ein differenzierbares Feld von selbstadjungierten $(1,1)-$ Tensoren über der vollständigen Riemannschen Mannigfaltigkeit $M$, dessen kovariantes Differential $D A$ verschwindet. Dann sind die Eigenwerte von $A$ konstant, die Eigendistributionen von $A$ integrabel und parallel und $M$ ist Riemannsches Produkt der Integralmannigfaltigkeiten dieser Eigendistributionen.

Die folgenden Sätze lassen sich mit geringem Mehraufwand für Immersionen in Räume konstanter Krümmung beweisen. Der Kürze halber sollen hier nur Immersionen in euklidische Räume betrachtet werden. Zur Methode für die Übertragung auf den allgemeineren Fall vergleiche man die Ausführungen in [10].

Satz 4. $x: M \rightarrow R^{N}$ sei eine isometrische Immersion der irreduziblen geschlossenen Riemannschen Mannigfaltigkeit $M$, deren Normalenbündel einen parallelen Schnitt $\xi$ mit $\operatorname{Det}\left(A_{\xi}\right) \neq 0$ und $\operatorname{Sp}\left(A_{\xi}^{-1}\right)=$ const. zuläßt. Die durch $\xi$ auf $M$ induzierte Metrik besitze nichtnegative Schnittkrümmung. Dann ist $x(M)$ in einer zu $\xi$ senkrechten Sphäre enthalten.

Beweis. Für die durch $\xi$ induzierte Metrik $\widetilde{<}, . \tilde{>}$ auf $M$ gilt wegen $\operatorname{Sp}\left(A_{\xi}^{-1}\right)$ $=\widetilde{\mathrm{Sp}}\left(A_{\xi}^{-1}\right)=$ const. und Lemma 2 nach (1.10) für $A_{\xi}^{-1}$

$$
\frac{1}{2} \tilde{\Delta} \operatorname{Sp}\left(A_{\xi}^{-2}\right)=\sum_{i<j} \tilde{K}_{i j}\left(\frac{1}{\lambda_{i}}-\frac{1}{\lambda_{j}}\right)^{2}+\tilde{\|} \tilde{D} A_{\xi}^{-1} \tilde{\|}^{2},
$$

$\left(\lambda_{i}=\right.$ Eigenwerte von $\left.A_{\xi}\right)$. Da die $\tilde{K}_{i j}$ voraussetzungsgemäß nicht negativ sind, ist die rechte Seite von (3.1) nicht negativ und verschwindet deshalb wegen der Kompaktheit von $M$ nach dem Lemma von Hopf identisch. Daraus folgt $\tilde{D} A_{\xi}^{-1}$ $=0$. Mit (2.2) erhält man dann $D A_{\xi}=0$. Wegen der Irreduzibilität von $M$ muß $A_{\xi}$ nach Lemma 3 zur Identität proportional sein, d.h., $\xi$ ist ein paralleler Schnitt im Normalenbündel von $x$, in dessen Richtung $x$ nabelsch ist. Nach einem Lemma von K. Nomizu (vgl. [5, Lemma 1]) ist $x$ deshalb in einer zu $\xi$ senkrechten Sphäre enthalten.

Bemerkung 4. Das Analogon zu Satz 4 für nichtnegative Schnittkrümmung von $M$ und $S p\left(A_{\xi}\right)=$ const. findet man bei B. Smyth [10]. Die Bedingung, daß die durch $\xi$ auf $M$ induzierte Metrik nichtnegative Schnittkrümmung besitzt, ist im vorliegenden irreduziblen Fall wegen (2.6) zur Definitheit von $A_{\xi}$ und zur nichtnegativen Schnittkrümmung der ursprünglichen Metrik auf $M$ äquivalent.

Ist $x: M \rightarrow M^{\prime}$ eine isometrische Immersion in einen Raum konstanter Krümmung, deren Normalenbündel lokal parallelisierbar ist, so erhält man durch Aufsummieren entsprechender Versionen von (1.2) für parallele lokale Schnitte 
im Normalenbündel von $x$ die zentrale Formel (Lemma 2.3) der Arbeit [12] von K. Yano und S. Ishihara. Für die dort abgeleiteten Sätze wird neben nichtnegativer Schnittkrümmung vorausgesetzt, daß die mittlere Krümmungsnormale $\eta$ von $x$ parallel ist, was $S p\left(A_{\eta}\right)=$ const. impliziert. Entsprechend der geometrischen Bedeutung von $\eta$ werden Charakterisierungen recht spezieller Immersionen erzielt. Wie man sich an Beispielen leicht verdeutlicht, müssen die Erwartungen bei Voraussetzung eines parallelen Normalvektorfeldes $\xi$ mit $\operatorname{Sp}\left(A_{\xi}\right)=$ const. reduziert werden.

Satz 5. $x: M \rightarrow R^{N}$ sei eine isometrische Immersion einer geschlossenen Riemannschen Mannigfaltigkeit nichtnegativer Schnittkrümmung. Das Normalenbündel von $x$ sei lokal parallelisierbar und lasse einen globalen parallelen Schnitt $\xi$ mit $\operatorname{Sp}\left(A_{\xi}\right)=$ const. zu. Dann ist $M$ Riemannsches Produkt $M_{1} \times$ $\cdots \times M_{k}$ Riemannscher Mannigfaltigkeiten $M_{i}$ und $x$ Produkt $x_{1} \times \cdots \times x_{k}$ isometrischer Immersionen $x_{i}: M_{i} \rightarrow R^{n_{i}}, R^{n_{1}} \times \cdots \times R^{n_{k}}=R^{N}$, wobei $k$ die Anzahl der verschiedenen Eigenwerte von $A_{\xi}$ angibt und die $x_{i}$ bis auf höchstens eine Ausnahme sphärisch sind.

Beweis. Aus der nichtnegativen Schnittkrümmung von $M$ und $\operatorname{Sp}\left(A_{\xi}\right)=$ const. folgt wie im Beweis von Satz 4 mit (1.10) $D A_{\xi}=0$. Sind $\lambda_{1}, \cdots, \lambda_{k}$ die verschiedenen Eigenwerte von $A_{\xi}$, so zerfällt $M$ deshalb nach Lemma 3 in ein Riemannsches Produkt $M_{1} \times \cdots \times M_{k}$, so daß ein beliebiger Tangentialvektor an den $i$-ten Faktor $M_{i}$ gerade Eigenvektor von $\boldsymbol{A}_{\xi}$ zum Eigenwert $\lambda_{i}$ ist. Da das Normalenbündel von $x$ lokal parallelisierbar ist, gilt für einen beliebigen Normalvektor $\xi^{\prime}$ in $p \in M$

$$
A_{\xi^{\prime}} \circ A_{\xi}=A_{\xi} \circ A_{\xi^{\prime}},
$$

(vgl. [12, Lemma 2.1]). Daraus folgt, daß die Eigenrichtungen von $A_{\xi \prime}$ Tangenten an einen der Faktoren von $M_{1} \times \cdots \times M_{k}$ sind. Aus Dimensionsgründen gilt deshalb für beliebige Tangentialvektoren $Y_{i}$ bzw. $Y_{j}$ an den $i$-ten bzw. $j$-ten Faktor von $M_{1} \times \cdots \times M_{k}$ in $p$

$$
\left\langle A_{\xi \prime}\left(Y_{i}\right), Y_{j}\right\rangle=0,
$$

falls $i \neq j$. Daraus schließt man leicht, daß $x$ Produkt $x_{1} \times \cdots \times x_{k}$ von isometrischen Immersionen $x_{i}: M_{i} \rightarrow R^{n_{i}}$ mit $R^{n_{1}} \times \cdots \times R^{n_{k}}=R^{N}$ ist (vgl. ein Lemma von J. D. Moore [7]). Ist der Eigenwert $\lambda_{i}$ von Null verschieden, so wird durch

$$
y_{i}(p)=x(p)-\lambda_{i}^{-1} \xi(p)
$$

eine differenzierbare Abbildung $y_{i}: M \rightarrow R^{N}$ definiert, die gerade die Integralmannigfaltigkeiten der zu $\lambda_{i}$ gehörigen Eigendistribution auf Punkte abbildet. Wegen der Parallelität von $\xi$ ist die Länge von $\xi$ konstant, womit gezeigt ist, daß $x_{i}$ sphärisch ist. 
Satz 6. $x: M \rightarrow R^{N}$ sei eine isometrische Immersion einer geschlossenen Riemannschen Mannigfaltigkeit nichtnegativer Schnittkrümmung. Das Normalenbündel von $x$ sei lokal parallelisierbar und lasse einen globalen parallelen Schnitt $\xi z u$, so daß $A_{\xi}$ definit und $\operatorname{Sp}\left(A_{\xi}^{-1}\right)$ konstant ist. Dann ist $M$ Riemannsches Produkt $M_{1} \times \cdots \times M_{k}$ und $x$ Produkt $x_{1} \times \cdots \times x_{k}$ isometrischer Immersionen $x_{i}$ in Sphären, wobei $k$ die Anzahl der verschiedenen Eigenwerte von $A_{\xi}$ angibt.

Beweis. Wegen $\operatorname{Sp}\left(A_{\xi}^{-1}\right)=$ const. gilt (2.8). Da $M$ nichtnegative Schnittkrümmung besitzt und $A_{\xi}$ definit ist, gilt

$$
\left(\lambda_{i} \lambda_{j}\right)^{-1} \cdot K_{i j} \geq 0 \text {. }
$$

Wie oben erhält man damit aus (2.8) $\tilde{D} A_{\xi}^{-1}=0$, woraus $D A_{\xi}=0$ folgt. Der Rest des Beweises verläuft dann analog zum Beweis von Satz 5 . Wegen der Definitheit von $A_{\xi}$ sind alle $x_{i}$ sphärisch.

Bemerkung 5. Analoge Aussagen über $x$ bekommt man, wenn man die lokale Parallelisierbarkeit des Normalenbündels von $x$ in den Voraussetzungen von Sätze 5 und 6 durch Beschränkungen der Kodimension von $x$ ersetzt, wie sie in [7] angegeben werden.

Folgerung 3. $\quad x: M \rightarrow R^{N}$ sei eine isometrische Immersion der geschlossenen 2-dimensionalen Mannigfaltigkeit $M$. Das Normalenbündel von $x$ lasse einen globalen parallelen Schnitt $\xi$ mit

$$
\operatorname{Det}\left(A_{\xi}\right) \neq 0, \quad \operatorname{Sp}\left(A_{\xi}^{-1}\right)=\text { const. }, \quad K \cdot \operatorname{Det}\left(A_{\xi}\right) \geq 0
$$

$z u$, wobei $K$ die Gaußsche Krümmung von $M$ bezeichnet. Dann ist a) $x(M)$ in einer zu $\xi$ senkrechten Sphäre enthalten oder b) $M$ ein flacher Torus $S^{1} \times S^{1}$ und $x$ ein Produkt sphärischer Kurven.

Beweis. Wegen $\left(\lambda_{1} \lambda_{2}\right)^{-1} K_{12}=\left(\operatorname{Det}\left(A_{\xi}\right)\right)^{-1} K \geq 0$ folgt aus (2.8) mit Lemma 3, daß die Eigenwerte $\lambda_{1}$ und $\lambda_{2}$ von $A_{\xi}$ konstant sind. Für $\lambda_{1}=\lambda_{2}$ folgt Teil a analog zum Beweis von Satz 4. Für $\lambda_{1} \neq \lambda_{2}$ muß $M$ nach Lemma 3 zum flachen Torus isometrisch sein. Ferner folgt aus der Parallelität von $\xi$ für einen beliebigen Normalvektor $\xi^{\prime}$ in $p \in M$

$$
A_{\xi^{\prime}} \circ A_{\xi}=A_{\xi} \circ A_{\xi^{\prime}} .
$$

Wegen der Verschiedenheit der Eigenwerte von $A_{\xi}$ erhält man daraus, daß das Normalenbündel von $x$ lokal parallelisierbar ist. Damit ist nach Satz 6 alles bewiesen.

Das Analogon zu Folgerung 3 für $S p\left(A_{\xi}\right)=$ const. ist in einem Spezialfall in [2] und im allgemeinen Fall in [11] enthalten. Es kann ebenso recht einfach aus Satz 5 gefolgert werden. 


\section{Literatur}

[ 1 ] S. Braidi \& C. C. Hsiung, Submanifolds of spheres, Math. Z. 115 (1970) 235-251.

[2] B. Y. Chen, A characterization of standard flat tori, Proc. Amer. Math. Soc. 37 (1973) 564-567.

[ 3 ] - Submanifolds in a Euclidean hypersphere, Proc. Amer. Math. Soc. 27 (1971) 627-628.

[ 4 ] S. S. Chern, M. do Carmo \& S. Kobayashi, Minimal submanifolds of a sphere with second fundamental form of constant length, Functional Analysis and Related Fields, Springer, Berlin, 1970, 60-75.

[ 5 ] D. Ferus, The torsion form of submanifolds in $\mathrm{E}^{\mathrm{N}}$, Math. Ann. 193 (1970) 114-120.

[ 6 ] S. Kobayashi \& K. Nomizu, Foundations of differential geometry, Vol. I, II, Interscience, New York, 1963, 1969.

[ 7 ] J. D. Moore, Isometric immersions of Riemannian products, J. Differential Geometry 5 (1971) 159-168.

[ 8 ] K. Nomizu \& B. Smyth, A formula of Simons' type and hypersurfaces with constant mean curvature, J. Differential Geometry 3 (1969) 367-377.

[9] J. Simons, Minimal varieties in Riemannian manifolds, Ann. of Math. 88 (1968) 62-105.

[10] B. Smyth, Submanifolds of constant mean curvature, Publikation in Vorbereitung.

[11] B. Wegner, Eine Charakterisierung von Produkten geschlossener sphärischer Kurven.

[12] K. Yano \& S. Ishihara, Submanifolds with parallel mean curvature vector, J. Differential Geometry 6 (1971) 95-118.

TECHNISCHE UNIVERSITÄT BERLIN 